\title{
Human-specific staphylococcal virulence factors enhance pathogenicity in a humanised zebrafish C5a receptor model.
}

Kyle D. Buchan ${ }^{\star 1,3,4}$, Michiel van Gent ${ }^{\star 2,5}$, Tomasz K. Prajsnar ${ }^{1}$, Nikolay V. Ogryzko ${ }^{1}$, Nienke W.M. de Jong ${ }^{2}$, Julia Kolata ${ }^{2}$, Simon J. Foster ${ }^{3}$, Jos A.G. van Strijp ${ }^{2}$, Stephen A. Renshaw ${ }^{\dagger 1}$

1. The Bateson Centre and Department of Infection, Immunity and Cardiovascular Disease, University of Sheffield, Western Bank, Sheffield, S10 2TN, UK.

2. Department of Medical Microbiology, University Medical Center Utrecht, Utrecht University, Utrecht, The Netherlands.

3. The Florey Institute for Host-Pathogen Interactions, Department of Molecular Biology and Biotechnology, University of Sheffield, Western Bank, Sheffield, S10 2TN, UK.

4. Current affiliation: Department of Biochemistry and Molecular Biology, University of Texas Medical Branch, Galveston, Texas, USA.

5. Current affiliation: Department of Microbiology, The University of Chicago, Chicago, USA.

Short title: Humanising zebrafish C5aR enhances S. aureus infection

${ }^{+}$Corresponding Author address:

The Bateson Centre

Firth Court

Western Bank

Sheffield

\section{S10 2TN}

Tel: +44 1142222334

Email: s.a.renshaw@sheffield.ac.uk 


\section{Abstract}

29 Staphylococcus aureus infects approximately $30 \%$ of the human population and causes a spectrum of pathologies ranging from mild skin infections to life-threatening invasive diseases.

31 The strict host specificity of its virulence factors has severely limited the accuracy of in vivo 32 models for the development of vaccines and therapeutics. To resolve this, we generated a 33 humanised zebrafish model and determined that neutrophil-specific expression of the human

34 C5a receptor conferred susceptibility to the $S$. aureus toxins PVL and HIgCB, leading to 35 reduced neutrophil numbers at the site of infection and increased infection-associated mortality as a direct result of the interaction between $S$. aureus and the receptor. These results show that humanised zebrafish provide a valuable platform to study the contribution of humanspecific S. aureus virulence factors to infection in vivo that could facilitate the development of novel therapeutic approaches and essential vaccines. 
Staphylococcus aureus is a highly specialised pathogen that colonises $30 \%$ of the human population and causes a variety of mild to severe illnesses ranging from skin and softtissue infections to necrotising pneumonia, endocarditis and septicaemia (1). In the USA, as many as $50 \%$ of $S$. aureus infections are caused by antibiotic-resistant strains (2), with methicillin-resistant $S$. aureus (MRSA) among the leading causes of death by a single infectious agent (3), emphasising the need for development of alternative therapies or vaccines. Despite promising results from vaccine studies utilising bacterial surface components and toxins as antigens, these candidates have largely failed to translate from traditional infection models to humans (4). A likely reason for this is the inability of current in vivo models to accurately recapitulate human infections, as $S$. aureus expresses a variety of strictly human-specific virulence factors that are ineffective in these models (4). Although their contribution to natural infection remains poorly understood, these virulence factors broadly target the host innate immune system to impair the complement system, oxidative enzymes, chemotactic proteins and phagocytic cells in order to evade recognition and destruction (5). To properly recapitulate human infections and to study the contributions of $S$. aureus virulence factors to infectivity and pathogenesis in vivo, a new humanised infection model is required.

Three S. aureus virulence factors interact with the human C5a receptor (C5AR1, CD88), a seven-transmembrane loop G-protein coupled receptor (GPCR) that is highly expressed on the surface of neutrophils (6) and recognises host anaphylatoxin C5a released during complement activation to control phagocyte activation and chemotaxis. The targeting of C5AR1 by multiple $S$. aureus virulence factors appears to be specifically focused against neutrophils, which form an essential line of defence against staphylococcal infection (7). The two bicomponent pore-forming toxins Panton-Valentine Leukocidin (PVL) and $\mathrm{y}$-Haemolysin $\mathrm{CB}(\mathrm{HlgCB})$ target $\mathrm{C} 5 \mathrm{AR} 1$ to recognise and lyse phagocytic cells by forming $\beta$-barrel pores in the cell membrane $(8,9)$. The toxins are secreted as two subunits, known as the S- (slow, 
LukS-PV/HIgC) and F- (fast, LukF-PV/HlgB) subunits due to their chromatography elution profiles. Besides inducing cell lysis, S-subunits also disrupt chemotaxis by competitively inhibiting receptor signalling $(8,9)$. In addition, the chemotaxis inhibitory protein of $S$. aureus (CHIPS) is a $14 \mathrm{kDa}$ protein that prevents C5a-mediated chemotaxis by binding directly to the $\mathrm{N}$-terminus of C5AR1 (10). The high-affinity protein-protein interactions between virulence factors and C5AR1, which have been characterised at the amino-acid level, are highly human specific and consequently our insight into the roles they play during infection is limited due to a lack of suitable humanised infection models $(4,11)$.

The zebrafish (Danio rerio) is a widely used model organism for investigating bacterial infections and the innate immune system $(12,13)$. Due to their optical transparency, ability to produce high numbers of offspring and genetic tractability, zebrafish offer many unique approaches over existing infection models. Zebrafish have a fully-developed innate immune system by two days post-fertilisation characterised by the presence of mature phagocytic cells (14) and a complement system that is highly homologous to humans (15).

In this study, we developed a humanised C5AR1 knock-in zebrafish infection model and determined the contribution of the $S$. aureus toxins PVL and HIgCB to infection in vivo. Whereas wild type zebrafish neutrophils were resistant to toxin-mediated lysis, we show that neutrophil-specific C5AR1 expression confers sensitivity of zebrafish neutrophils to PVL and HIgCB-mediated lysis in vivo. Humanised zebrafish displayed reduced neutrophil abundance at the sites of infection and increased $S$. aureus-associated mortality as a result of the direct interaction between $S$. aureus and the human C5a receptor when expressed by zebrafish neutrophils. In conclusion, our studies not only illustrate the critical contribution of PVL and HIgCB to in vivo infection and pathogenesis, but also show the significance of humanised zebrafish as a novel platform to investigate the activities of human-specific virulence factors in vivo and to accurately recapitulate natural human infection in a model organism. 


\section{Zebrafish possess a functional $\mathrm{C} 5 \mathrm{aR}$ that is responsive to serum-derived $\mathrm{C5a}$}

To study the C5a-C5aR signalling axis in zebrafish, we first expressed the zebrafish

C5a receptor (c5ar1) in the human monocyte-like cell line U937 (U937-dreC5aR) by lentiviral

transduction and measured the receptor's ability to bind and respond to recombinant zebrafish and human $\mathrm{C5a}$ (dreC5a and hsaC5a, respectively) using previously established methods (8).

As controls, we generated cells stably expressing the human C5a receptor (U937-hsaC5aR), or an empty vector control (U937-EV). Firstly, flow-cytometric analysis of recombinant, FITClabelled C5a-binding capacities showed that U937-dreC5aR cells specifically bound dreC5a, but not hsaC5a (Fig. 1A). Conversely, U937-hsaC5aR cells strongly bound hsaC5a and only minimally interacted with dreC5a, suggesting that both receptors bind $\mathrm{C} 5 \mathrm{a}$ in a speciesspecific manner. Activation of GPCRs, including the C5a receptor, results in the induction of intracellular signalling cascades culminating in the cytosolic release of intracellular calcium stores (16). Accordingly, we evaluated the signalling ability of the receptors by measuring intracellular $\mathrm{Ca}^{2+}$ release following receptor stimulation using the Fluo-3AM probe. Treatment of U937-dreC5aR cells with recombinant dreC5a provoked robust calcium release, indicating successful receptor ligation and activation, whereas U937-hsaC5aR expressing cells responded minimally to dreC5a (Fig. 1B). Importantly, zymosan-activated zebrafish serum induced a response similar to recombinant $\mathrm{C} 5 \mathrm{a}$, indicating that the zebrafish $\mathrm{C} 5 \mathrm{aR}$ responds to physiological concentrations of zebrafish $\mathrm{C} 5 \mathrm{a}$ in activated fish serum under these conditions (Fig. 1B). Conversely, U937-hsaC5aR cells responded robustly to hsaC5a or human activated

114 serum treatment, but not dreC5a treatment. Taken together, these data show that the 115 zebrafish c5ar1 gene encodes a functional surface receptor that interacts with, and is activated 116 by, physiological concentrations of C5a. Furthermore, the human and zebrafish C5a receptor 117 and ligand pairs are species specific and are not interchangeable. 


\section{Zebrafish C5aR is resistant to human-specific virulence factors}

119 The three S. aureus C5AR1-targeting virulence factors, CHIPS, PVL, and HIgCB, are known 120 to display strict human specificity and are unable to interact with C5aRs expressed by several 121 other species $(8,9)$. However, their ability to target zebrafish complement components is 122 unknown. To test this, we first assessed the functionality of the zebrafish C5a receptor in the absence and presence of the inhibitory protein CHIPS. We observed that U937-dreC5aR cells retained complete responsiveness to dreC5a in the presence of CHIPS at concentrations that completely inhibited U937-hsaC5aR cells, indicating that CHIPS is ineffective at targeting the zebrafish receptor (Fig. 1C). Based on our detailed molecular understanding of the interactions between CHIPS and the 21-amino acid binding site in the $\mathrm{N}$-terminus of the human C5aR, we predicted that a few amino-acid changes may be sufficient to render the zebrafish C5aR sensitive to the inhibitory actions of CHIPS. To test this, we generated ten rationally designed variants of the zebrafish receptor, substituting human residues at key points within the CHIPS-binding site (17). All ten variants showed normal surface expression and endogenous signalling activity in response to recombinant dreC5a similar to the wildtype zebrafish receptor (Fig. S1). Notably, activation of three partly humanised variants (Fig. S2, variants $\mathbf{A}, \mathbf{H}$ and I) by dreC5a was effectively inhibited in the presence of CHIPS, the most conservative change requiring only three amino acid substitutions (Fig. S2, variant $\mathbf{H}$ ). These data indicated that sensitisation of dreC5aR to CHIPS can be achieved with only three amino acid changes in the endogenous receptor.

Next, we determined the sensitivity of zebrafish C5aR-expressing cells to poreformation and lysis by the $S$. aureus toxins PVL and HlgCB. Whereas U937-hsaC5aR cells were efficiently permeabilised in the presence of the recombinant leukocidin components of

141 PVL or HIgCB, U937-dreC5aR cells were resistant to these same concentrations of PVL and

142 HlgCB (Fig. 1D,E). We then aimed to identify the minimal amino-acid changes in the zebrafish

143 C5aR that are sufficient to gain sensitivity to PVL and/or HlgCB using the same strategy as 144 we used to gain CHIPS sensitivity. We tested an extensive set of dreC5aR variants with many 
145 combinations of (partly) humanised intracellular and extracellular loops and individual amino

146 acids that are known to be involved in the PVL/HlgCB interaction with the hsaC5aR.

147 Unfortunately, we failed to achieve toxin-sensitivity while maintaining surface expression and

148 signalling capability of the dreC5aR. In conclusion, we found that the wild type zebrafish C5aR

149 is insensitive to the lytic activity of $S$. aureus virulence factors CHIPS, PVL, and HIgCB at the

150 concentrations tested. While CHIPS sensitivity can be achieved with only three amino-acid 151 changes in dreC5aR, we were unable to identify dreC5aR variants that gained PVL and/or 152 HlgCB sensitivity.

154 Generation of transgenic zebrafish with neutrophil specific human C5AR1 expression

Next, we sought to develop an in vivo infection model to study the role of $S$. aureus pore-forming toxins during natural infection. Because we were unable to generate a humanised drC5aR sensitive to PVL and/or HlgCB in vitro, we instead introduced the complete human C5a receptor into zebrafish neutrophils. To this end, a transgenic construct directing expression of a fluorescent, Clover-tagged C5AR1 driven by the zebrafish neutrophil-specific lyz promoter (18) was introduced into the zebrafish genome by Tol2 transgenesis (19), producing the transgenic line $T g(l y z$ hsaC5AR1-Clover)sh505. To verify whether C5AR1Clover expression was restricted to zebrafish neutrophils, we crossed this line to the established transgenic zebrafish line $\operatorname{Tg}(\operatorname{lyz}: n f s B-m C h e r r y) s h 260$ that displays neutrophilspecific mCherry expression (18). In the double-transgenic larvae, we observed Clover expression exclusively in the mCherry-positive cells, confirming that the C5AR1 protein is expressed specifically in zebrafish neutrophils (Fig. 2A,B). Furthermore, whereas mCherry showed general cytoplasmic localisation, the C5AR1-associated Clover signal was enriched at the cell membrane of neutrophils from $\operatorname{Tg}(l y z: h s a C 5 a R 1-C l o v e r) s h 505$ zebrafish, 
171

172

173

174

175

176

177

178

179

180

181

182

183

184

185

186

187

188

189

190

191

192

193

194

195

correctly recapitulated in the humanised zebrafish system (Fig. 2C,D). Notably, the total number of neutrophils in these fish was unaffected by transgene expression (Fig. S3).

\section{Human C5AR1 is functional in humanised zebrafish}

Next, we investigated functional activity of human C5AR1 in the transgenic zebrafish line by assessing neutrophil migration to recombinant dreC5a and hsaC5a injected into the otic vesicles, two sac-like invaginations in the head of the fish that are a preferred site for assessing phagocyte migration (20). In non-humanised lyz:nfsB-mCherry fish, dreC5a injection resulted in migration of neutrophils to the injection site, as expected due to endogenous receptor function, while hsaC5a injection did not induce neutrophil migration (Fig. 3A,B). In contrast, neutrophils expressing the human C5AR1 transgene displayed robust migration towards the site of hsaC5a injection, showing that C5AR1 acts as a functional C5a receptor in zebrafish neutrophils that is able to direct neutrophil migration.

\section{Humanised zebrafish neutrophils are targeted by PVL and HIgCB in vivo}

Having established that human C5AR1 is expressed as a functional receptor on the surface of zebrafish neutrophils, we next investigated whether C5AR1-expressing neutrophils are targeted by the C5AR1-targeting $S$. aureus toxins PVL and HIgCB in vivo. To this end, the community-acquired MRSA strain USA300 was injected with or without recombinant PVL into the otic vesicle of wild type or C5AR1-transgenic larvae, and the number of neutrophils present at the injection site was determined 4 hours later. Whereas injection of USA300 alone resulted in similar numbers of neutrophils at the injection site in both C5AR1 negative and positive larvae, the addition of PVL significantly reduced neutrophil numbers specifically in C5AR1expressing larvae, while not affecting neutrophil migration in C5AR1-negative fish (Fig. 4A,B). Similarly, we observed a reduced number of neutrophils at the injection site in humanised 
larvae injected with USA300 together with HIgCB (Fig. 4C,D). These results showed that C5AR1 expression sensitises zebrafish neutrophils to PVL and HIgCB in vivo. signalling at low concentrations $(8,9)$. However, HlgC-only injection did not significantly affect neutrophil abundance at the injection site, suggesting that the reduced neutrophil counts observed in the presence of HlgCB injection are due to pore-forming activity of HlgCB and cell lysis rather than inhibition of $\mathrm{C} 5 \mathrm{aR}$ signalling by $\mathrm{HlgC}$ alone (Fig. 4C,D). In conclusion, our data indicated that human C5AR1 expression in zebrafish neutrophils conferred sensitivity to the $S$. aureus toxins PVL and HlgCB, and showed that the presence of these toxins reduces neutrophil numbers at the sites of infection in vivo.

\section{Zebrafish expressing human C5AR1 are more susceptible to $S$. aureus infection}

Given that human C5AR1 acted as a functional receptor that was targeted by the $S$. aureus toxins PVL and HIgCB in our zebrafish model, we next sought to determine whether neutrophil-specific expression of C5AR1 increases the susceptibility of humanised fish to staphylococcal infection. To investigate this, we first separated the fish into non-humanised (lyz:nfsB-mCherry only) and humanised (lyz:hsaC5AR1-Clover; lyz:nfsB-mCherry) groups and injected the community-acquired MRSA strain USA300 into the circulation valley of the fish at $30 \mathrm{hpf}$ according to previously published methods (21). In this model, macrophages are able to clear $S$. aureus from the fish circulation, so to specifically study the effect of neutrophils, we silenced irf8 expression using an irf8 morpholino to alter zebrafish haematopoiesis and

217 favour the differentiation of neutrophils over macrophages (22). In this way, we observed 218 significantly higher mortality following staphylococcal infection for the C5AR1-positive 219 zebrafish compared to the C5AR1-negative fish, both when infected at 30hpf or 50hpf (Fig. $5 \mathrm{~A}, \mathbf{B})$. This demonstrates that expression of human C5AR1 in zebrafish neutrophils enhances susceptibility to staphylococcal infection and suggests that the C5aR-targeting toxins PVL and HlgCB play crucial roles in determining the severity of $S$. aureus infection. 
Complete understanding of staphylococcal infection requires a thorough characterisation of its virulence factors and an appreciation of their physiological relevance and synergistic role during natural infection. Due to a lack of appropriate animal models, human-specific virulence factors have been difficult to adequately study in vivo, creating a gap in our understanding that has disrupted the experimental validation of effective $S$. aureus vaccine candidates and novel therapeutic approaches. In this study, we addressed this problem by generating a humanised zebrafish model that allowed us to study the important contributions of human-specific $S$. aureus toxins PVL and HIgCB to infection-related mortality in a relevant in vivo system.

S. aureus expresses three virulence factors that target the human complement receptor $\mathrm{C5aR}$, which is highly expressed on the surface of human neutrophils. Although components from all arms of the complement system (classical, alternative, lectin) have been found in larval zebrafish and are known to confer early humoral immunity to the embryo via maternal transfer (23-25), the zebrafish complement system including C5aR has barely been characterised at the functional level. To facilitate our studies of the C5aR-targeting virulence factors, we first established functionality of the C5a-C5aR axis in zebrafish. By expressing the zebrafish C5aR on U937 cells we showed that dreC5aR ligation by recombinant zebrafish C5a or zymosan-activated zebrafish serum resulted in similar $\mathrm{Ca}^{2+}$ mobilization as observed upon 244 activation of the human receptor. Furthermore, we observed neutrophil migration toward the sites of dreC5a injection in vivo. Together, these results suggest that the C5a-C5aR axis is functional in zebrafish and, analogous to the human situation, is involved in directing neutrophil migration towards invasive pathogens. Interestingly, both human and zebrafish C5a displayed strict species specificity and were not interchangeable with one another. We further found that, in agreement with studies in other species (8-10), S. aureus virulence factors CHIPS, PVL, 
and $\mathrm{HlgCB}$ were ineffective against the zebrafish $\mathrm{C} 5 \mathrm{aR}$ receptor, corroborating their strict human specificity. suggests that inhibition of neutrophil function is an important contributor to $S$. aureus infection success. Neutrophils play an essential role in protecting the body from acute bacterial infection, and play a prominent role in clearing $S$. aureus infections ( 7 . Importantly, the $S$. aureus toxins are thought to exacerbate infection-associated morbidity and mortality (26), however, due to the well-characterised human specificity of the interaction between C5aR and these toxins, their contribution to infection in vivo has remained elusive. The introduction of the human C5aR into zebrafish neutrophils allowed us to study the contribution of PVL and HlgCB-mediated targeting of the C5aR on neutrophils during natural infection and with highthroughput. In this way, we found that the actions of both toxins result in reduced neutrophil presence at the site of infection and increased infection-associated mortality. The presence of HlgC alone, which was recently shown to inhibit neutrophil chemotaxis (9), did not affect neutrophil numbers, suggesting that it is the cytotoxic activity of these toxins that leads to reduced neutrophil numbers in these fish, and not a blockade of neutrophil migration. infections. Uniquely, in zebrafish systemic infection is primarily controlled by macrophages, while neutrophils are recruited almost solely to surface-associated infections (27). To mimic human infections more closely and to study the role of neutrophils specifically, we utilised the irf8 morpholino, which causes macrophages to undergo a cell-fate switch by suppression of the irf8 transcription factor (22). This depletes macrophages and promotes differentiation into

272 neutrophils, producing neutrophil-replete zebrafish larvae that can be used to assess the role 273 of toxin-mediated virulence in a way that is more representative of a human $S$. aureus 274 infection. Neutrophil-replete zebrafish expressing the human C5aR were significantly more 275 susceptible to $S$. aureus infection compared with C5AR1-negative siblings both in fish infected from 30 hours post-fertilisation (hpf) and also from 50 hours post-fertilisation. In both cases, 
277 S. aureus infection produces significant mortality from as early as 20 hours post-infection and 278 is significantly enhanced by the expression of the human C5aR. Interestingly, fish infected at $27950 \mathrm{hpf}$ appear to be twice as susceptible to those infected at $30 \mathrm{hpf}$, accounting for a $40 \%$ and $20 \%$ increase in mortality respectively compared with C5AR1-negative siblings. This could be the result of an increased number of neutrophils at 50hpf compared with $30 \mathrm{hpf}(14)$, suggesting that mortality is further enhanced by the direct interaction between hC5aRexpressing neutrophils and human-adapted virulence factors.

Our work shows that selective humanisation of the zebrafish is a powerful approach towards identifying the contribution of host-restricted virulence to bacterial infection and pathogenesis. Interestingly, several other $S$. aureus virulence factors target GPCRs. For example, CXCR2 is targeted by SSL5, Staphopain A, and Leukotoxin ED (28-30) and the formyl peptide receptors (FPR1, FPR2) are targeted by CHIPS, FLIPr and SSL13 (31-33). We anticipate that more extensive humanisation of these and other receptors in zebrafish will lead to infection models that incorporate a multitude of human-specific virulence factors and even more closely resemble human infections, permitting detailed investigation of the interplay and relative importance of these virulence factors at the difference stages of infection. Our ultimate goal is to only marginally alter the endogenous zebrafish receptors at specific amino acids to minimally interfere with the zebrafish physiology. Our detailed knowledge of the receptor interaction sites will be harnessed to design minimally humanised receptors that gain susceptibility to the $S$. aureus virulence factors while maintaining in vivo functionality. Unfortunately, we were so far unable to generate a partially humanised zebrafish C5aR that displayed sensitivity to the pore-forming toxins and was still functionally expressed at the cell surface. Amino-acid substitutions in the extracellular loops of the receptor quickly abolished surface expression and thereby led to non-functional receptors, forcing us to introduce the entire human receptor into zebrafish neutrophils. It is promising however that only three aminoacid changes appeared sufficient to confer CHIPS-mediated inhibition to an otherwise functional receptor in vitro. 
305 contribution of human-specific S. aureus virulence factors to infection outcome in vivo.

306 Importantly, we believe that this model provides a starting point that can be further developed

307 to incorporate additional human-specific virus-host interactions, functioning as an improved,

308 expandable, and translatable platform to accurately assess the efficacy of $S$. aureus-targeted 309 therapeutic interventions. 
Cells, Lentiviral transductions

314 Type Culture Collection) and grown in RPMI or DMEM medium, respectively, supplemented with glutamine, penicillin/streptomycin and 10\% FBS. C5AR1 (CD88; NM_001736) and C5aR (XM_005159274) were cloned into a dual promoter lentiviral vector (BIC-PGK-Zeo-T2amAmetrine; RP172) described elsewhere (34). This vector expresses the cloned transgene

318 from an EF1A promoter as well as the fluorescent protein mAmetrine and the selection marker

319 ZeoR from the PGK promoter. Third generation lentiviral particles were produced in HEK293T cells following standard lentivirus production methods. Spin infection of U937 cells was performed by adding $100 \mu \mathrm{l}$ virus supernatant with $8 \mu \mathrm{g} / \mathrm{mL}$ polybrene to 50.000 cells and spinning at $1000 \mathrm{~g}$ for $2 \mathrm{~h}$ at $33^{\circ} \mathrm{C}$. Transduced cell lines were selected to high purity (>95\%) by selection with $400 \mu \mathrm{g} / \mathrm{ml}$ Zeocin starting two days post transduction, and transgene expression was confirmed by flow cytometry using a mouse anti-FLAG M2 antibody (F1804,

Sigma-Aldrich) together with an APC or PE-conjugated secondary anti-mouse antibody (Jackson) and acquisition on a FACSCantoll (BD Bioscience) cytometer. For expression of the zebrafish $\mathrm{C5a}$ receptor the full-length mRNA coding sequence was used (XM_005159274.1).

\section{C5aR-signalling assay} human serum albumin (HSA) at room temperature under constant agitation for 10 min, then washed and suspended to 3 million cells per $\mathrm{mL}$ in RPMI/0.05\% HSA followed by data acquisition on a FACSVERSE (BD Biosciences). Basal fluorescence level for each sample 
continuing the acquisition to measure signalling-induced cytosolic $\mathrm{Ca}^{2+}$ release by increased

337 Fluo-3AM fluorescence.

\section{Flow cytometry analysis of C5a binding}

Binding of FITC-labelled hsaC5a (human C5a) or dreC5a (zebrafish C5a) was determined by incubating U937 cells with $10 \mu \mathrm{g} / \mathrm{mL}$ FITC-C5a in RPMI supplemented with $0.05 \%$ HSA for 30 minutes on ice. After washing the samples were analysed on a FACSVERSE (BD Biosciences).

\section{Collection of zebrafish serum}

Zebrafish blood was kindly supplied by Dr. Astrid van der Sar (Amsterdam University

Medical Centre) and serum collection was performed following a previously published protocol

348 (35). Subsequently, 10\% serum in HEPES buffer was incubated with zymosan for 30 minutes at $37^{\circ} \mathrm{C}$ to activate the alternative complement pathway that results in $\mathrm{C} 5 \mathrm{a}$ generation. The activated serum was centrifuged at $10,000 \mathrm{rpm}$ and the supernatant containing the anaphylatoxins was stored at $-80^{\circ} \mathrm{C}$.

\section{Cell permeability assays}

Cells were resuspended in $100 \mu \mathrm{L} \mathrm{RPMI} / 0.05 \% \mathrm{HSA}$ and incubated with for $30 \mathrm{~min}$ at $37^{\circ} \mathrm{C}$ with $10 \mu \mathrm{g} / \mathrm{mL}$ PVL or HIgCB (as PVL and HlgCB are two-component toxins, equimolar concentrations of polyhistidine-tagged LukS-PV, LukF-PV, HlgC and HlgB were used). Cells were then stained with $1 \mu \mathrm{g} / \mathrm{mL}$ 4',6-diamidino-2-phenylindole (DAPI; Molecular Probes/Thermo Fisher) and analysed on a FACSVERSE (BD Biosciences). Pore formation was defined as the percentage of cells positive to DAPI staining. 
361

362

363

364

365

366

367

368

369

370

371

372

373

374

375

376

377

378

379

380

381

382

383

384

385

\section{Recombinant protein production and FITC labelling}

LukS-PV, LukF-PV, HIgC and HIgB were cloned and expressed as previously described $(8,9,36)$. From the coding sequence of zebrafish C5 (XM_001919191.5) we identified a predicted C5a cleavage product (KFE DKA QKY GAF REY CLS GTR SSP TLE TCK DRA NRV TLP NKK TRR DYE KEK YCR LAF EQC CVF AKD LRK E) and included nine additional amino acids from an alignment with human C5 (NAE NII LSR). This sequence was codon-optimised for expression in E. coli K-12 and ordered as a gBlock (Integrated DNA Technologies). This was then ligated into the BamHI and Notl sites of the modified expression vector pRSETB (Invitrogen Life Technologies), containing a cleavable N-terminal polyhistidine tag and three glycines (6xHis-TEV-GGG-dreC5a) or a non-cleavable N-terminal polyhistidine tag. Zebrafish C5a was expressed in E. coli strain Rosetta-gami(DE3)pLysS (Novagen; Merck Biosciences). Following cell lysis with $10 \mu \mathrm{g} / \mathrm{mL}$ lysozyme and three freezethaw sonication cycles in $20 \mathrm{mM}$ sodium phosphate $(\mathrm{pH} 7.8)$, the His-tagged proteins were purified using nickel-affinity chromatography (HiTrap chelating, HP; GE Healthcare) with an imidazole gradient (10-250 mM; Sigma-Aldrich). Purified protein was stored in PBS at $-20^{\circ} \mathrm{C}$. Subsequently, the polyhistidine tag of $6 x$ His-TEV-dreC5a was removed by incubation with TEV protease (Thermo Fisher Scientific) and the protein was FITC-labelled at the N-terminus using the sortagging method (37). CHIPS protein was purified using previously published methods $(32,38)$.

\section{Zebrafish Husbandry}

Zebrafish (Danio rerio) were raised and maintained according to standard protocols (39) in UK Home Office-approved aquaria at the Bateson Centre, University of Sheffield, and kept under a $14 / 10$ hour light/dark regime at $28^{\circ} \mathrm{C}$. 


\section{Creation of Tg(lyz:hsaC5AR1-Clover)sh505 zebrafish}

The plasmid used for introducing the transgene into the zebrafish genome (pDestTol2CG2 lyz:hsaC5AR1-Clover cmlc2:eGFP) was created by Gateway cloning (19).

The C5AR1 gene was PCR amplified from the pIRES-C5AR1 plasmid with a truncated stop codon to allow C' terminal fusion of clover and ligated into the middle-entry clone vector pDONR221. The final construct was created by an LR reaction combining a 5' vector containing the lyz promoter, the middle entry vector pDONR221 C5AR1-Clover, a 3' vector containing the Clover fluorophore, and the destination vector pDestTol2CG2. To induce transgenesis, plasmid DNA of pDestTol2CG2 lyz:hsaC5AR1-Clover cmlc2:eGFP was injected into zebrafish embryos at the one-cell stage with $10 \mathrm{ng} / \mu \mathrm{l}$ of Tol2 transposase mRNA, according to published protocols (39). At three days post-fertilisation, positive transgenic larvae were selected and raised to maturity, then screened for successful germline integration of the construct.

\section{Injection into the zebrafish otic vesicle}

1\% agarose dishes supplemented with E3 were cast using triangular moulds. Before

402 injection, larvae were anaesthetised by immersion in $0.02 \%(w / v)$ Tricaine prior to transfer to 403 the mounting dish. Larvae were then arranged laterally in rows. Excess media was then removed with a pipette to minimise movement during injection. Larvae were then injected from the dorsal side into the otic vesicle. Four hours after injection, larvae were fixed for 1 hour in 4\% paraformaldehyde, and later stained with Sudan Black B to indicate neutrophils (performed according to published methods (18)). Proteins were injected at the highest available concentration, which were $10 \mu \mathrm{M}$ and $90 \mu \mathrm{M}$ for hsaC5a (human) and dreC5a (zebrafish) respectively, and $30.3 \mu \mathrm{M}$ and $16.7 \mu \mathrm{M}$ for $\mathrm{PVL}$ and HIgCB respectively. 
411 Systemic infection of zebrafish embryos and irf8 knockdown

412 Zebrafish larvae at 30 or $50 \mathrm{hpf}$ were microinjected into the circulation with bacteria as 413 previously described (21). Briefly, anaesthetised larvae were embedded in $3 \% \mathrm{w} / \mathrm{v}$ 414 methylcellulose and injected individually with $1 \mathrm{nl}$ using microcapillary pipettes filled with the 415 bacterial suspension of known concentration. Following infection, larvae were observed 416 frequently up to $122 \mathrm{hpf}$ and numbers of dead larvae recorded at each time point. Morpholino417 modified antisense oligomer against and irf8 (splice MO) (22) was injected using a method 418 described previously (21).

\section{Bacterial Culture Preparation}

To prepare a liquid overnight culture of $S$. aureus, $5 \mathrm{ml}$ of $\mathrm{BHI}$ broth medium (Oxoid) 422 was inoculated with a colony of $S$. aureus strain USA300 and incubated at $37^{\circ} \mathrm{C}$ overnight with shaking. To prepare $S$. aureus for injection, $50 \mathrm{ml}$ of $\mathrm{BHI}$ media was inoculated with $500 \mu \mathrm{l}$ of overnight culture and incubated for roughly 2 hours at $37^{\circ} \mathrm{C}$ with shaking. The $\mathrm{OD}_{600}$ of each culture was measured and $40 \mathrm{ml}$ of the remaining culture harvested by centrifugation at $4500 \mathrm{~g}$ for 15 minutes at $4^{\circ} \mathrm{C}$. The pellet was then resuspended in a volume of PBS appropriate to the bacterial dose required. Once the pellets were resuspended they were then kept on ice until required.

\section{Statistics}

All data were analysed in Prism 7.0 (GraphPad Software, San Diego, CA, USA).

432 Comparisons between groups were performed using a two-way ANOVA with different multiple 433 comparisons tests depending on whether the group was compared with a control (Dunnett's 434 test) or not (Bonferroni's test). Significance was assumed at $p<0.05$. 
1. S. Y. C. Tong, J. S. Davis, E. Eichenberger, T. L. Holland, V. G. Fowler, Staphylococcus aureus infections: Epidemiology, pathophysiology, clinical manifestations, and management. Clin. Microbiol. Rev. 28, 603-661 (2015).

2. G. J. Moran, A. Krishnadasan, R. J. Gorwitz, G. E. Fosheim, L. K. McDougal, R. B. Carey, D. A. Talan, EMERGEncy ID Net Study Group, Methicillin-resistant S. aureus infections among patients in the emergency department. N. Engl. J. Med. 355, 666-74 (2006).

3. R. M. Klevens, M. A. Morrison, J. Nadle, S. Petit, K. Gershman, S. Ray, L. H. Harrison,

4. W. Salgado-Pabón, P. M. Schlievert, Models matter: the search for an effective Staphylococcus aureus vaccine. Nat. Rev. Microbiol. 12, 585-591 (2014).

5. K. D. Buchan, S. J. Foster, S. A. Renshaw, Staphylococcus aureus: Setting its sights on the human innate immune system. Microbiol. (United Kingdom). 165 (2019), doi:10.1099/mic.0.000759.

6. Y. Laumonnier, C. M. Karsten, J. Köhl, Novel insights into the expression pattern of anaphylatoxin receptors in mice and men. Mol. Immunol., 0-1 (2017).

7. A. N. Spaan, B. G. J. Surewaard, R. Nijland, J. a G. van Strijp, Neutrophils versus Staphylococcus aureus: a biological tug of war. Annu. Rev. Microbiol. 67, 629-50 (2013).

8. A. N. Spaan, T. Henry, W. J. M. van Rooijen, M. Perret, C. Badiou, P. C. Aerts, J. Kemmink, C. J. C. de Haas, K. P. M. van Kessel, F. Vandenesch, G. Lina, J. A. G. van Strijp, The Staphylococcal Toxin Panton-Valentine Leukocidin Targets Human C5a Receptors. Cell Host Microbe. 13, 584-594 (2013). Benito, C. J. C. de Haas, C. J. Day, M. P. Jennings, G. Lina, F. Vandenesch, K. P. M. van Kessel, V. J. Torres, J. A. G. van Strijp, T. Henry, The staphylococcal toxins Yhaemolysin $\mathrm{AB}$ and $\mathrm{CB}$ differentially target phagocytes by employing specific chemokine receptors. Nat. Commun. 5, 5438 (2014). 
10. P.-J. Haas, C. J. C. de Haas, W. Kleibeuker, M. J. J. G. Poppelier, K. P. M. van Kessel, J. A. W. Kruijtzer, R. M. J. Liskamp, J. A. G. van Strijp, N-Terminal Residues of the Chemotaxis Inhibitory Protein of Staphylococcus aureus Are Essential for Blocking Formylated Peptide Receptor but Not C5a Receptor. J. Immunol. 173, 5704-5711 (2004).

11. V. G. Fowler, R. A. Proctor, Where does a Staphylococcus aureus vaccine stand? Clin. Microbiol. Infect. 20, 66-75 (2014).

12. K. M. Henry, C. a Loynes, M. K. B. Whyte, S. a Renshaw, Zebrafish as a model for the study of neutrophil biology. J. Leukoc. Biol. 94, 633-42 (2013).

13. V. Torraca, S. Mostowy, Zebrafish Infection: From Pathogenesis to Cell Biology. Trends Cell Biol. 28, 143-156 (2018).

14. G. J. Lieschke, A. C. Oates, M. O. Crowhurst, A. C. Ward, J. E. Layton, Morphologic and functional characterization of granulocytes and macrophages in embryonic and adult zebrafish. Blood. 98, 3087-96 (2001).

15. S. Zhang, P. Cui, Complement system in zebrafish. Dev. Comp. Immunol. 46, 3-10 (2014).

16. M. Y. Tawk, G. Zimmermann-Meisse, J. Bossu, C. Potrich, T. Bourcier, M. Dalla Serra, B. Poulain, G. Prévost, E. Jover, Internalization of staphylococcal leukotoxins that bind and divert the $\mathrm{C} 5 \mathrm{a}$ receptor is required for intracellular $\mathrm{Ca}(2+)$ mobilization by human neutrophils. Cell. Microbiol. 17, 1241-57 (2015).

17. B. Postma, W. Kleibeuker, M. J. J. G. Poppelier, M. Boonstra, K. P. M. Van Kessel, J. a G. Van Strijp, C. J. C. de Haas, Residues 10-18 within the C5a receptor $\mathrm{N}$ terminus compose a binding domain for chemotaxis inhibitory protein of Staphylococcus aureus. J. Biol. Chem. 280, 2020-7 (2005).

18. K. D. Buchan, T. K. Prajsnar, N. V Ogryzko, N. W. M. de Jong, M. van Gent, J. Kolata, S. J. Foster, J. A. G. van Strijp, S. A. Renshaw, A transgenic zebrafish line for in vivo visualisation of neutrophil myeloperoxidase. PLoS One. 14, e0215592 (2019).

19. K. M. Kwan, E. Fujimoto, C. Grabher, B. D. Mangum, M. E. Hardy, D. S. Campbell, J. M. Parant, H. J. Yost, J. P. Kanki, C. Bin Chien, The Tol2kit: A multisite gateway-based construction Kit for Tol2 transposon transgenesis constructs. Dev. Dyn. 236, 30883099 (2007).

20. E. L. Benard, A. M. van der Sar, F. Ellett, G. J. Lieschke, H. P. Spaink, A. H. Meijer, 
500

501

502

503

504

505

506

507

508

509

510

511

512

513

514

515

516

517

518

519

520

521

522

523

524

525

526

527

528

529

530

531

Infection of zebrafish embryos with intracellular bacterial pathogens. J. Vis. Exp., 1-8 (2012).

21. T. K. Prajsnar, V. T. Cunliffe, S. J. Foster, S. a Renshaw, A novel vertebrate model of Staphylococcus aureus infection reveals phagocyte-dependent resistance of zebrafish to non-host specialized pathogens. Cell. Microbiol. 10, 2312-25 (2008).

22. L. Li, H. Jin, J. Xu, Y. Shi, Z. Wen, Irf8 regulates macrophage versus neutrophil fate during zebrafish primitive myelopoiesis. Blood. 117, 1359-1369 (2011).

23. Z. Wang, S. Zhang, Z. Tong, L. Li, G. Wang, Maternal Transfer and Protective Role of the Alternative Complement Components in Zebrafish Danio rerio. PLoS One. 4, e4498 (2009).

24. L. Yang, L. Bu, W. Sun, L. Hu, S. Zhang, Functional characterization of mannosebinding lectin in zebrafish: implication for a lectin-dependent complement system in early embryos. Dev. Comp. Immunol. 46, 314-22 (2014).

25. A. M. Earley, C. L. Graves, C. E. Shiau, Critical Role for a Subset of Intestinal Macrophages in Shaping Gut Microbiota in Adult Zebrafish. Cell Rep. 25, 424-436 (2018).

26. T. S. Naimi, K. H. Ledell, K. Como-sabetti, S. M. Borchardt, D. J. Boxrud, S. K. Johnson, S. Fridkin, C. O. Boyle, R. N. Danila, Comparison of Community- and Health CareAssociated Staphylococcus aureus Infection. J. Am. Med. Assoc. 290, 2976-2984 (2003).

27. E. Colucci-Guyon, J.-Y. Tinevez, S. a Renshaw, P. Herbomel, Strategies of professional phagocytes in vivo: unlike macrophages, neutrophils engulf only surfaceassociated microbes. J. Cell Sci. 124, 3053-9 (2011).

28. J. Bestebroer, K. P. M. van Kessel, H. Azouagh, A. M. Walenkamp, I. G. J. Boer, R. A. Romijn, J. A. G. van Strijp, C. J. C. de Haas, Staphylococcal SSL5 inhibits leukocyte activation by chemokines and anaphylatoxins. Blood. 113, 328-37 (2009).

29. A. J. Laarman, G. Mijnheer, J. M. Mootz, W. J. M. van Rooijen, M. Ruyken, C. L. Malone, E. C. Heezius, R. Ward, G. Milligan, J. A. G. van Strijp, C. J. C. de Haas, A. R. Horswill, K. P. M. van Kessel, S. H. M. Rooijakkers, Staphylococcus aureus Staphopain A inhibits CXCR2-dependent neutrophil activation and chemotaxis. EMBO J. 31, 3607-3619 (2012).

30. T. Reyes-Robles, F. Alonzo, L. Kozhaya, D. B. Lacy, D. Unutmaz, V. J. Torres, 
Staphylococcus aureus Leukotoxin ED Targets the Chemokine Receptors CXCR1 and CXCR2 to Kill Leukocytes and Promote Infection. Cell Host Microbe. 14, 453-459 (2013).

31. Y. Zhao, K. P. M. van Kessel, C. J. C. de Haas, M. R. C. Rogers, J. A. G. van Strijp, P.J. A. Haas, Staphylococcal superantigen-like protein 13 activates neutrophils via formyl peptide receptor 2. Cell. Microbiol. 20, e12941 (2018).

32. C. J. C. de Haas, K. E. Veldkamp, A. Peschel, F. Weerkamp, W. J. B. Van Wamel, E. C. J. M. Heezius, M. J. J. G. Poppelier, K. P. M. Van Kessel, J. A. G. van Strijp, Chemotaxis Inhibitory Protein of Staphylococcus aureus, a Bacterial Antiinflammatory Agent. J. Exp. Med. 199, 687-695 (2004).

33. C. Prat, J. Bestebroer, C. J. C. de Haas, J. A. G. van Strijp, K. P. M. van Kessel, A New Staphylococcal Anti-Inflammatory Protein That Antagonizes the Formyl Peptide Receptor-Like 1. J. Immunol. 177, 8017-8026 (2006).

34. A. T. Tromp, M. Van Gent, P. Abrial, A. Martin, J. P. Jansen, C. J. C. De Haas, K. P. M. Van Kessel, B. W. Bardoel, E. Kruse, E. Bourdonnay, M. Boettcher, M. T. McManus, C. J. Day, M. P. Jennings, G. Lina, F. Vandenesch, J. A. G. Van Strijp, R. Jan Lebbink, P. A. Haas, T. Henry, A. N. Spaan, Human CD45 is an F-component-specific receptor for the staphylococcal toxin Panton-Valentine leukocidin. Nat. Microbiol. 3, 708-717 (2018).

35. G. L. Pedroso, T. O. Hammes, T. D. C. Escobar, L. B. Fracasso, L. F. Forgiarini, T. R. da Silveira, Blood Collection for Biochemical Analysis in Adult Zebrafish. J. Vis. Exp., 10-12 (2012).

36. M. Perret, C. Badiou, G. Lina, S. Burbaud, Y. Benito, M. Bes, V. Cottin, F. Couzon, C. Juruj, O. Dauwalder, N. Goutagny, B. A. Diep, F. Vandenesch, T. Henry, Cross-talk between Staphylococcus aureus leukocidins-intoxicated macrophages and lung epithelial cells triggers chemokine secretion in an inflammasome-dependent manner. Cell. Microbiol. 14, 1019-1036 (2012).

37. M. W. Popp, J. M. Antos, G. M. Grotenbreg, E. Spooner, H. L. Ploegh, Sortagging: A versatile method for protein labeling. Nat. Chem. Biol. 3, 707-708 (2007).

38. P. J. Haas, C. J. C. De Haas, M. J. J. C. Poppelier, K. P. M. Van Kessel, J. A. G. Van Strijp, K. Dijkstra, R. M. Scheek, H. Fan, J. A. W. Kruijtzer, R. M. J. Liskamp, J. Kemmink, The structure of the C5a receptor-blocking domain of chemotaxis inhibitory protein of Staphylococcus aureus is related to a group of immune evasive molecules. 
bioRxiv preprint doi: https://doi.org/10.1101/2020.02.18.955021; this version posted June 20, 2020. The copyright holder for this preprint (which was not certified by peer review) is the author/funder. All rights reserved. No reuse allowed without permission.

565 J. Mol. Biol. 353, 859-872 (2005).

566

39

C. Nüsslein-Volhard, R. Dahm, Zebrafish: A Practical Approach (Oxford; New York:

567 Oxford University Press, c2002., Practical., 2002).

568 
571 General: Thank you to C. Loynes and D. Drew for their training, advice and technical help.

572 Also, we'd like to thank the Bateson Centre aquarium staff for all their help, A. M. van der Sar 573 for facilitating the zebrafish blood collection, and the Wolfson Light Microscopy Facility.

574 Funding: This work was supported by AMR cross-council funding from the MRC to the 575 SHIELD consortium "Optimising Innate Host Defence to Combat Antimicrobial Resistance" 576 (MRNO2995X/1), a Medical Research Council (MRC), United Kingdom, Programme Grant 577 (MR/M004864/1) to S.A.R., and the University of Sheffield 2022 Futures programme via the 578 Florey Institute. T.K.P. was supported by an individual Marie Curie fellowship (PIEF-GA-2013579 625975). Author Contributions: K.D.B and M.v.G performed experiments with assistance 580 from T.K.P, N.V.O, N.W.M.d.J, and J.K.; S.A.R, J.A.G.v.S and S.J.F conceived the study and 581 designed experiments. T.K.P performed survival experiments on irf8 morphants. N.V.O 582 generated the Tg(lyz:nfsB-mCherry)sh260 line. K.D.B, M.v.G and S.A.R wrote the manuscript 583 with significant input from all authors. Competing Interests: The authors declare no conflicts of interest. Data and Materials Availability: The raw data for this manuscript is available at Mendeley Data, DOI: 10.17632/x55r5h6j4g.2.

586

Abbreviations: BHI Brain-Heart Infusion, C5aR C5a receptor, C5AR1 Human C5a receptor, c5ar1 Zebrafish C5a receptor, cfu colony forming units, CHIPS Chemotaxis inhibitory protein of Staphylococcus, dpf days post fertilisation, dpi days post injection, dreC5a Zebrafish C5a, EV Empty Vector, FLIPr FPRL1 Inhibitory Protein, FPR Formyl Peptide Receptor, GPCR G-Protein Coupled Receptor, HIgCB y-Haemolysin CB, hpf hours post fertilisation, hpi hours post injection, hsaC5a Human C5a, irf8 Interferon Regulatory Factor 8, MO Morpholino, MRSA Methicillin-Resistant Staphylococcus aureus, nl Nanolitre, $\mathbf{O D}_{600}$ Optical Density at 600 nanometres, PBS Phosphate- 
A
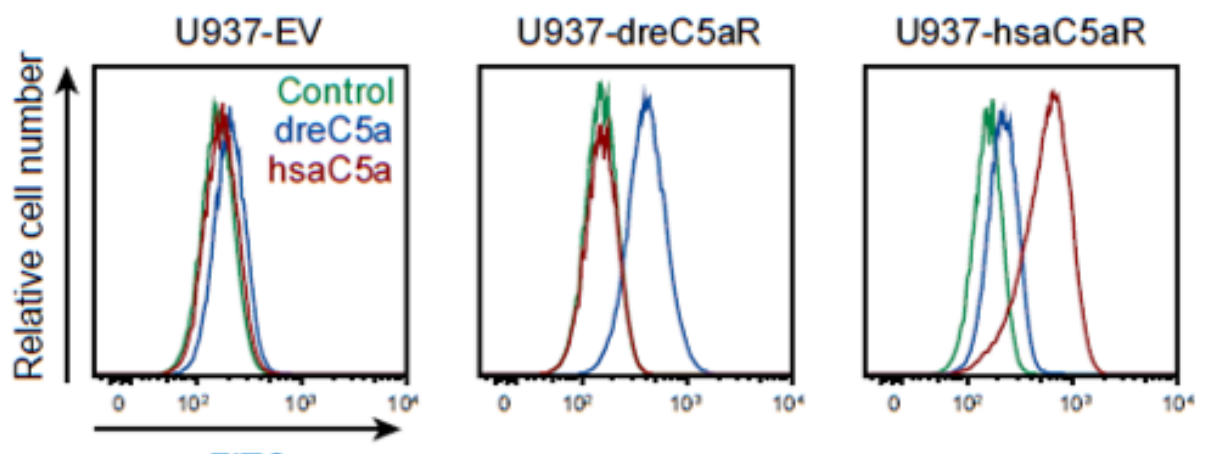

B
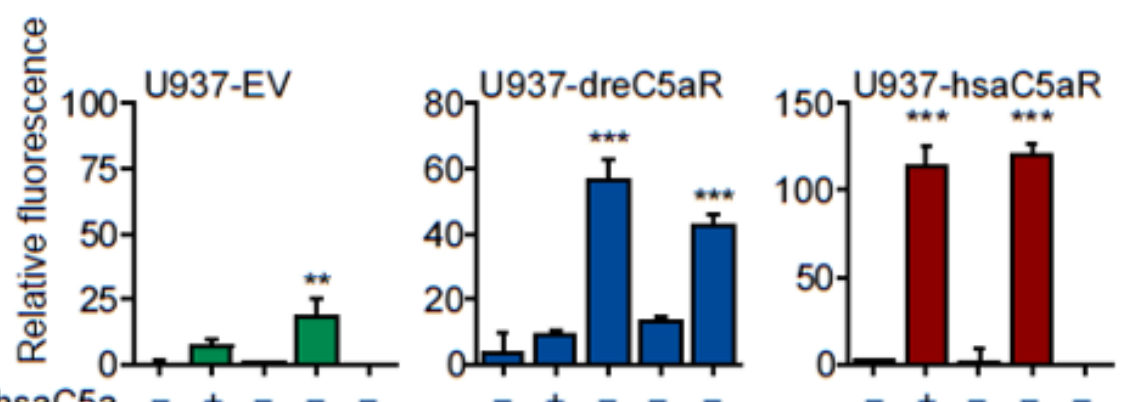

rec hsaC5a

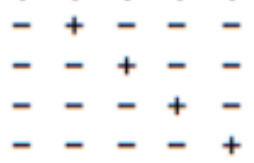

C

U937-dreC5aR
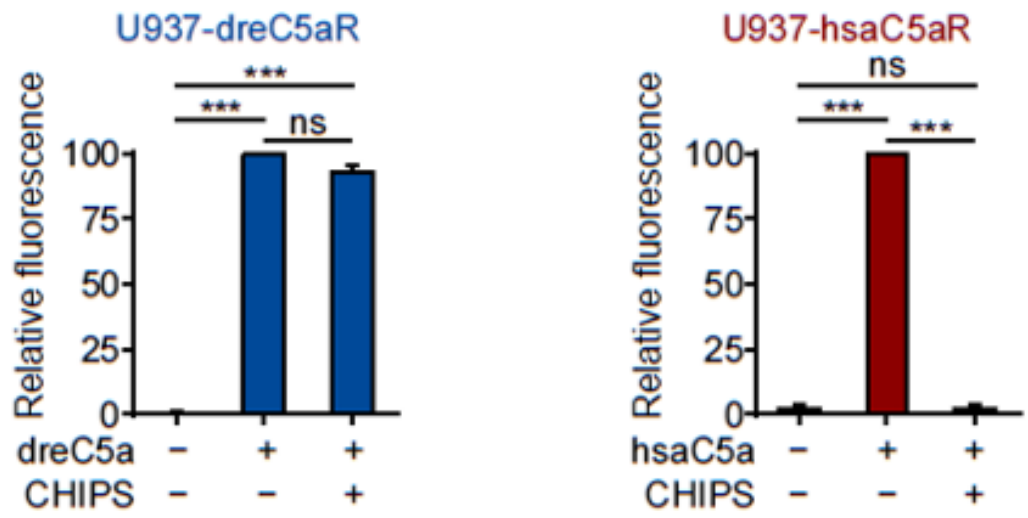

D

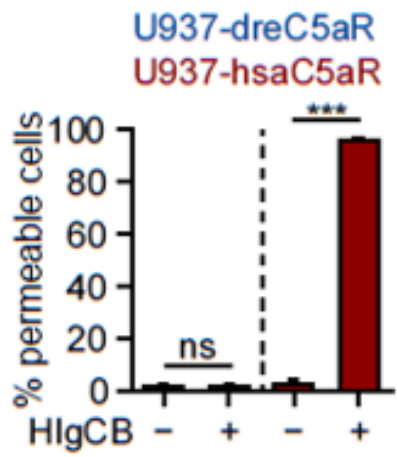

E

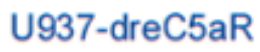

U937-hsaC5aR

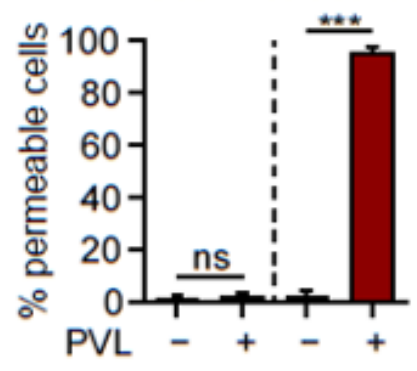


599 Figure 1. Zebrafish possess a functional C5aR that is insensitive to targeting by $S$. 600 aureus virulence factors CHIPS, PVL, and HIgCB.

601 A) Binding of FITC-labelled zebrafish C5a (dreC5a) or human C5a (hsaC5a) to U937 cells 602 stably expressing the zebrafish C5a receptor (dreC5aR), the human C5a receptor (hsaC5aR) or an empty vector control (EV) was determined by flow cytometry. B) Relative C5aR activity

604 following treatment with recombinant human or zebrafish C5a or zymosan-activated serum 605 was determined by measuring cytosolic calcium release using a $\mathrm{Ca}^{2+}$-sensitive fluorescent 606 probe (Fluo-3AM) and displayed as a percentage relative to the positive control (10 $\mu \mathrm{M}$ 607 ionomycin treatment, set at $100 \%$ ). C) C5aR activity following treatment with recombinant 608 human or zebrafish C5a with or without $10 \mu \mathrm{g} / \mathrm{ml}$ recombinant CHIPS was determined by 609 measuring cytosolic $\mathrm{Ca}^{2+}$ release using a $\mathrm{Ca}^{2+}$-sensitive fluorescent probe (Fluo-3AM) by flow

610 cytometry and displayed as mean percentage \pm SD relative to the C5a-treated sample without 611 CHIPS (middle bar, set at 100\%). D,E) Pore formation in U937-dreC5aR (blue) or U937612 hsaC5aR (red) cells following 30 min incubation with $10 \mu \mathrm{g} / \mathrm{ml} \mathrm{HlgCB}$ (D) or $10 \mu \mathrm{g} / \mathrm{ml} \mathrm{PVL} \mathrm{(E),}$ 613 as measured by percentage DAPI-positive cells by flow cytometry. Data are presented as 614 means $\pm \mathrm{SD}$; ns, not significant; ${ }^{*}, p<0.05 ;{ }^{* *}, p<0.01 ;{ }^{* \star *}, p<0.001$; two-way ANOVA with 615 Dunnett's multiple comparisons correction of each sample versus the untreated control (A-C) 616 or $t$-test $(\mathrm{D}, \mathrm{E})$. 
A

Tg(lyz:hsaC5AR1-Clover)sh505 x Tg(lyz:nfsB-mCherry)sh260

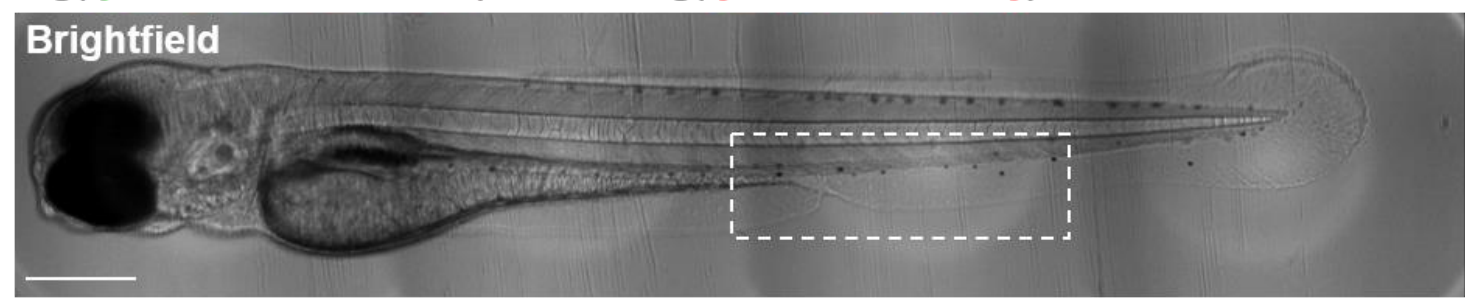

B
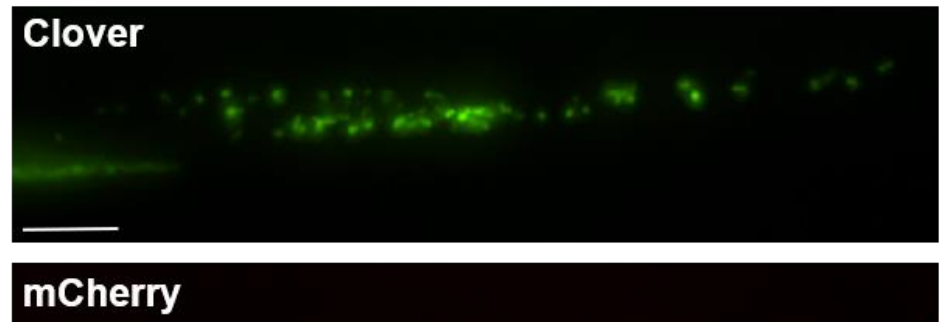

a

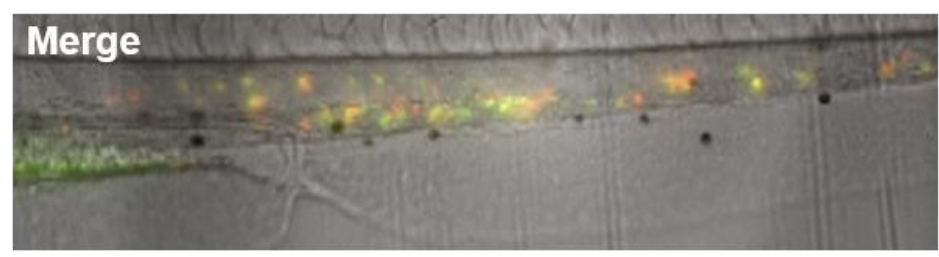

C

Tg(lyz:hsaC5AR1-Clover)sh505 x Tg(lyz:nfsB-mCherry)sh260
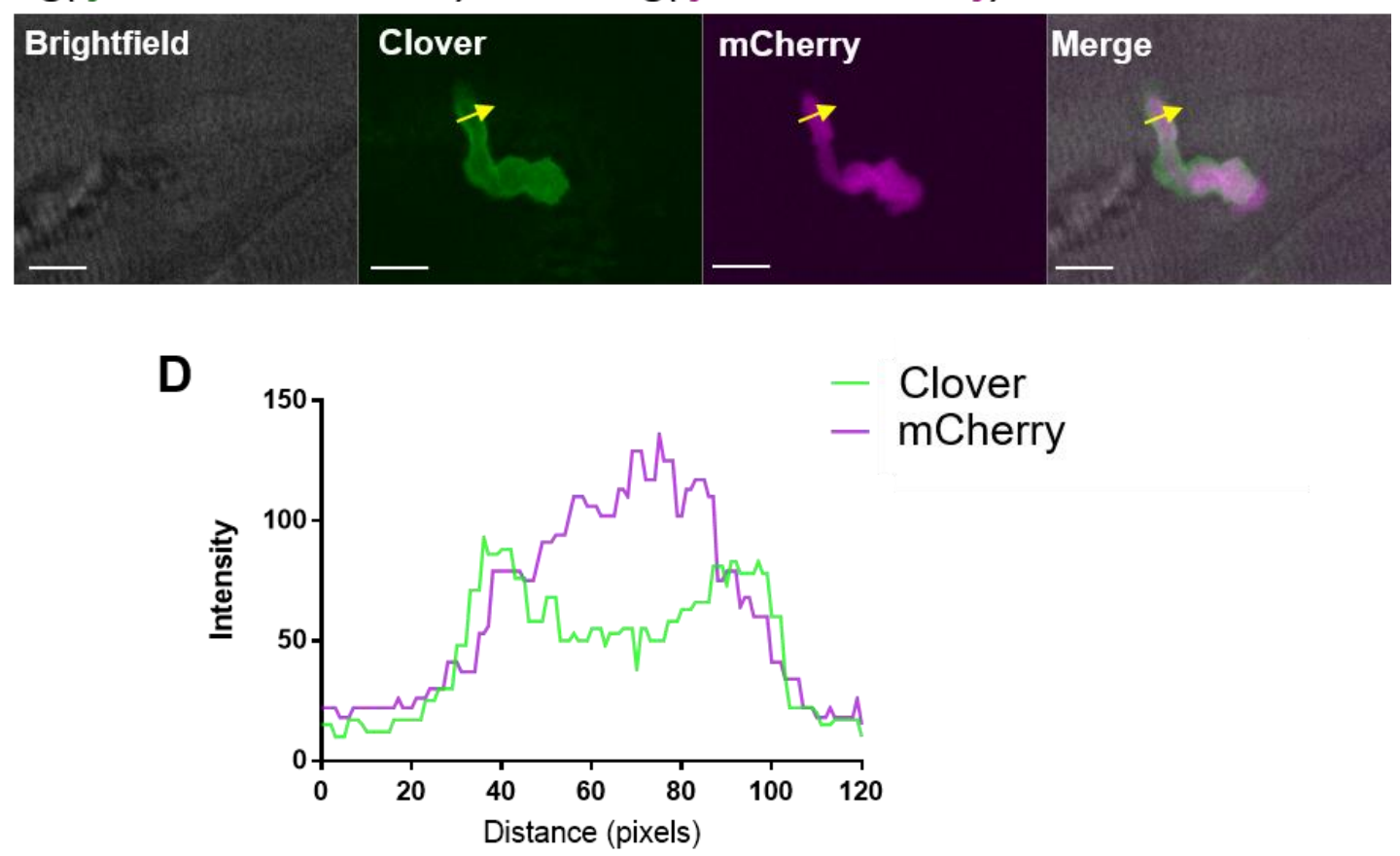
619 Figure 2. Generation of a transgenic zebrafish expressing human C5AR1-Clover.

620 A) A double-transgenic $T g$ (lyz:hsaC5AR1-Clover)sh505; Tg(lyz:nfsB-mCherry)sh260 larva at 621 3dpf. Dashed white box indicates the enlarged region shown in (B), scale bar $=250 \mu \mathrm{m}$. B)

622 Microscopy analysis of neutrophil-specific mCherry (red) and Clover (green) transgene 623 expression in the enlarged view of the caudal haematopoietic tissue shown in $(A)$, scale bar $=$ $624100 \mu \mathrm{m}$. C) Close-up of Clover (green) and mCherry (purple) expression in a double-transgenic 625 neutrophil in the caudal haematopoietic tissue of a 3dpf larva, scale bar $=10 \mu \mathrm{m}$. D) Line 626 intensity profile of the fluorescent signal of Clover (green) and mCherry (purple) across the 627 yellow arrow shown in (C).

628 
A
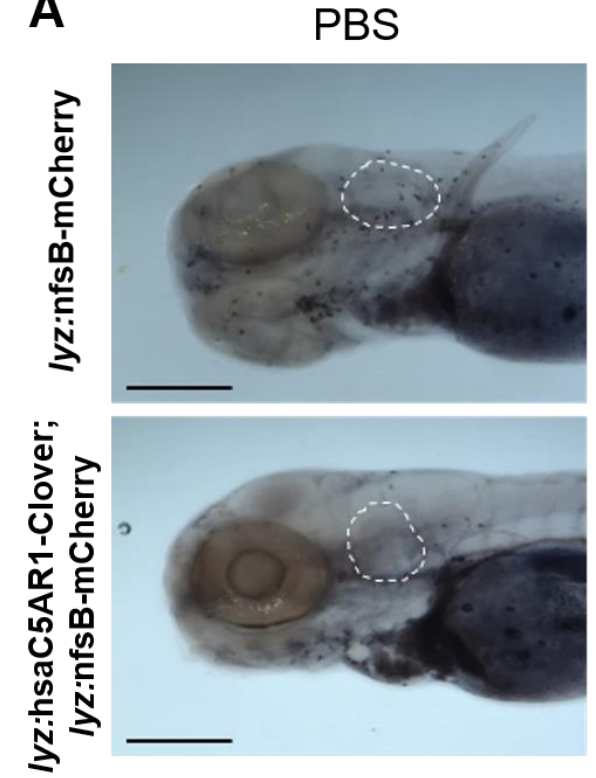

dreC5a
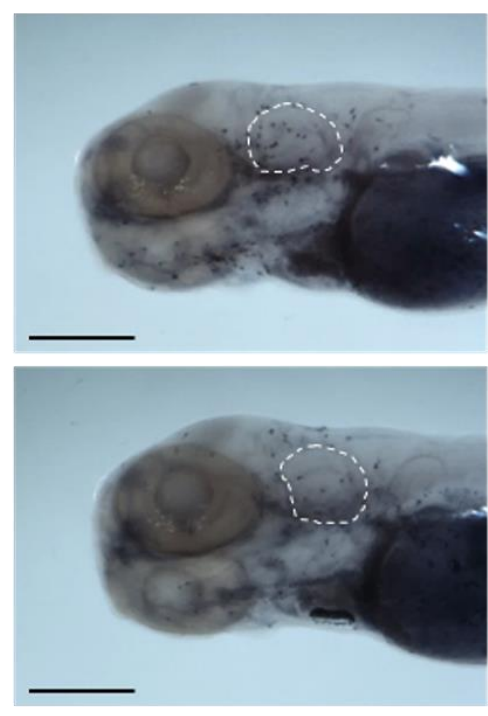

hsaC5a
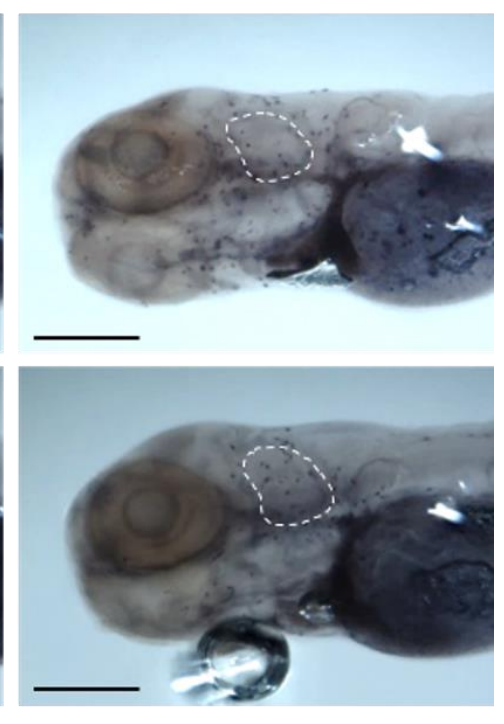

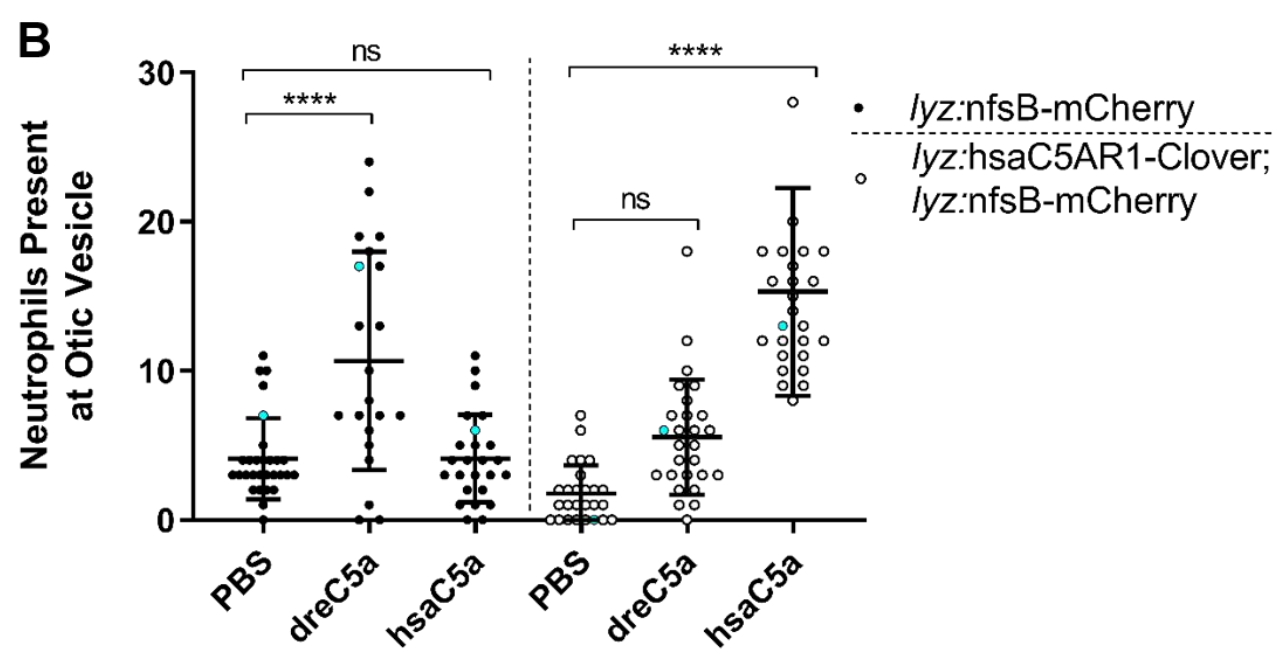

629

630 Figure 3. Human C5AR1 confers sensitivity to hsaC5a in humanised zebrafish.

631 A) Neutrophil migration was assessed in non-humanised (lyz:nfsB-mCherry) and humanised

632 (lyz:hsaC5AR1-Clover; lyz:nfsB-mCherry) zebrafish larvae at 3dpf following injection with a 633 PBS vehicle control, recombinant zebrafish C5a (dreC5a) or human C5a (hsaC5a) into the 634 otic vesicle. Four hours post injection (hpi), larvae were fixed in $4 \%$ paraformaldehyde and stained with Sudan Black B to detect neutrophils; scale bar $=200 \mu \mathrm{m}$. B) Numbers of

636 neutrophils present at the otic vesicle at $4 \mathrm{hpi}$ in zebrafish treated as in (A), blue points denote 637 the representative images in (A). Error bars shown are mean $\pm S D(n=22-26$ individual animals 
bioRxiv preprint doi: https://doi.org/10.1101/2020.02.18.955021; this version posted June 20, 2020. The copyright holder for this preprint (which was not certified by peer review) is the author/funder. All rights reserved. No reuse allowed without permission.

638 over two independent experiments); groups were analysed using a two-way ANOVA and 639 adjusted using Bonferroni's multiple comparisons test. ns, not significant; ${ }^{* * *}, p<0.0001$.

640 


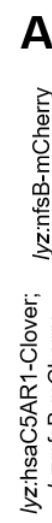
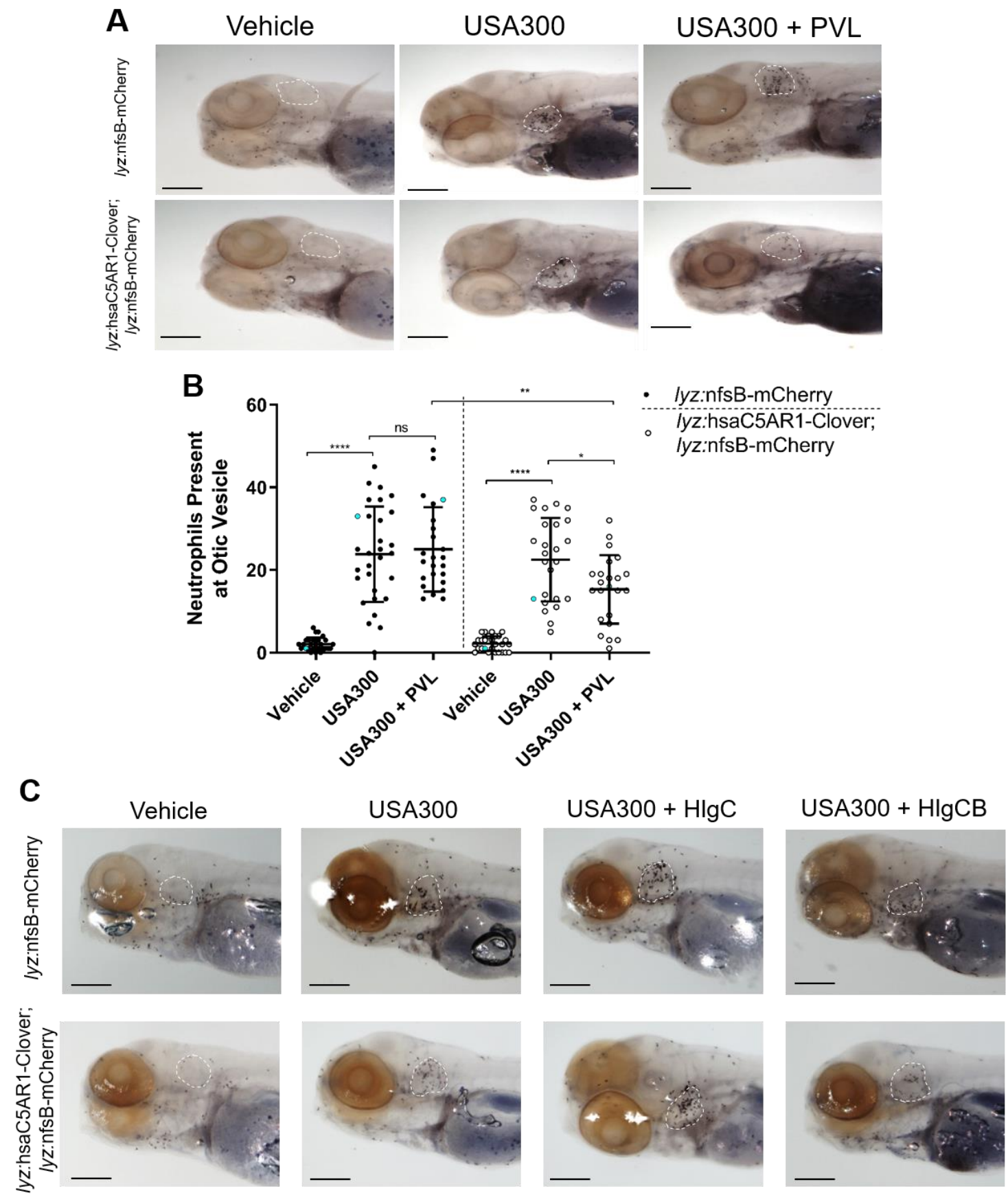

D

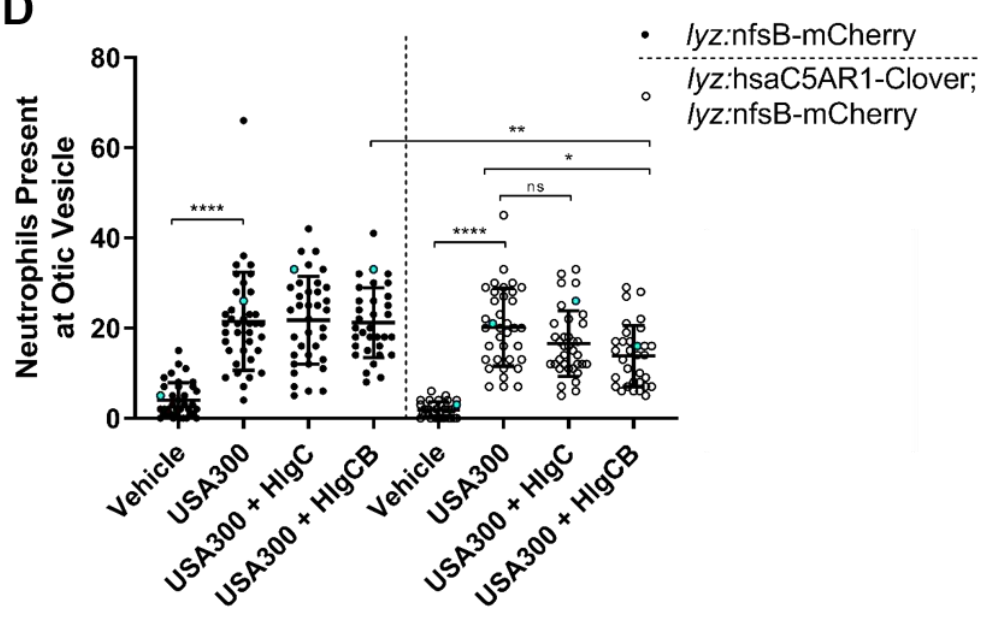


642 Figure 4. Neutrophils of C5AR1-expressing zebrafish are susceptible to $S$. aureus 643 toxins PVL and HIgCB.

644 A) Representative images of neutrophil abundance in zebrafish larvae at 3 dpf that were 645 separated into non-humanised (lyz:nfsB-mCherry only) and humanised (lyz:hsaC5AR1-

646 Clover; lyz:nfsB-mCherry) groups and injected into the otic vesicle with a vehicle control or $647 \sim 3,500 \mathrm{cfu}$ of $S$. aureus USA300 with or without $30.3 \mu \mathrm{g} / \mathrm{ml} \mathrm{PVL}$. The white outline indicates

648 the otic vesicle; scale bar $=200 \mu \mathrm{m}$. B) Number of neutrophils present at the otic vesicle at $6494 \mathrm{hpi}$, blue points denote the representative images in $(\mathrm{A})$. C) Representative images of 650 neutrophil abundance in zebrafish larvae injected into the otic vesicle as in $(A)$ with a vehicle 651 control, or $\sim 3,500$ S. aureus USA300 with or without $16.7 \mu \mathrm{g} / \mathrm{ml} \mathrm{HlgCB}$ or $\mathrm{HlgC}$, as indicated.

652 D) Number of neutrophils present at the otic vesicle at $4 \mathrm{hpi}$, blue points denote the 653 representative images in (C). Error bars shown are mean $\pm S D$, groups were analysed using 654 a two-way ANOVA and adjusted using Bonferroni's multiple comparisons test. (B) $n=22-26$ 655 over two independent experiments and (D) n=32-41 over three independent experiments. ns, 656 not significant; ${ }^{*}, p<0.05 ;{ }^{* *}, p<0.01 ;{ }^{* * *}, p<0.0001$. Scale bars $=200 \mu \mathrm{m}$. 
A

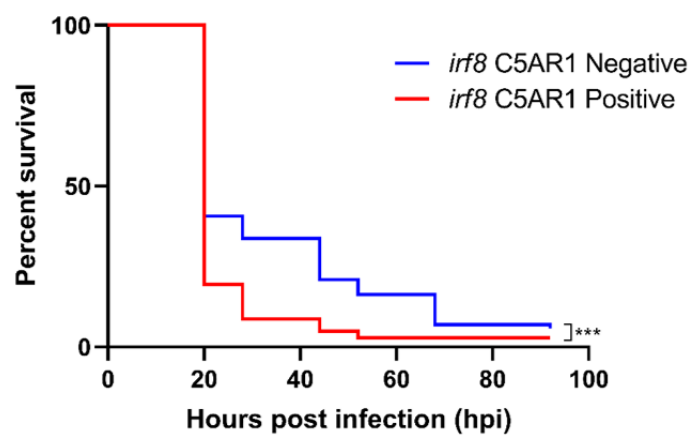

B

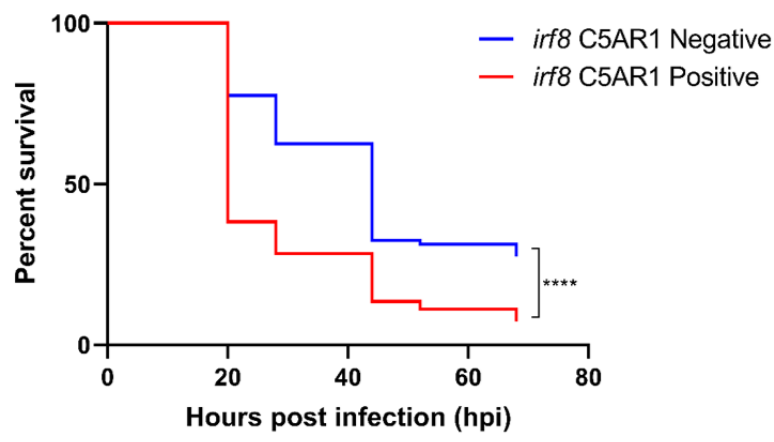

659 Figure 5. Humanised neutrophil-replete zebrafish are more susceptible to 660 staphylococcal infection.

661 Neutrophil-replete zebrafish were generated by injecting an irf8 morpholino at the single-cell 662 stage, silencing irf8 expression. Zebrafish larvae were then separated into non-humanised 663 (lyz:nfsB-mCherry only) and humanised (Iyz:hsaC5AR1-Clover; lyz:nfsB-mCherry) groups 664 and infected with A) 30hpf ( 700cfu), or B) 50hpf ( 2,000cfu). Survival was monitored up to 66596 hrs post infection and data was analysed using a Log-rank Mantel-Cox test. (A) $n=80-81$

666 over three independent experiments; ${ }^{* * *}, p<0.001$. (B) $n=86-103$ over three independent 667 experiments; ${ }^{* * * *}, p<0.0001$. 
670

671

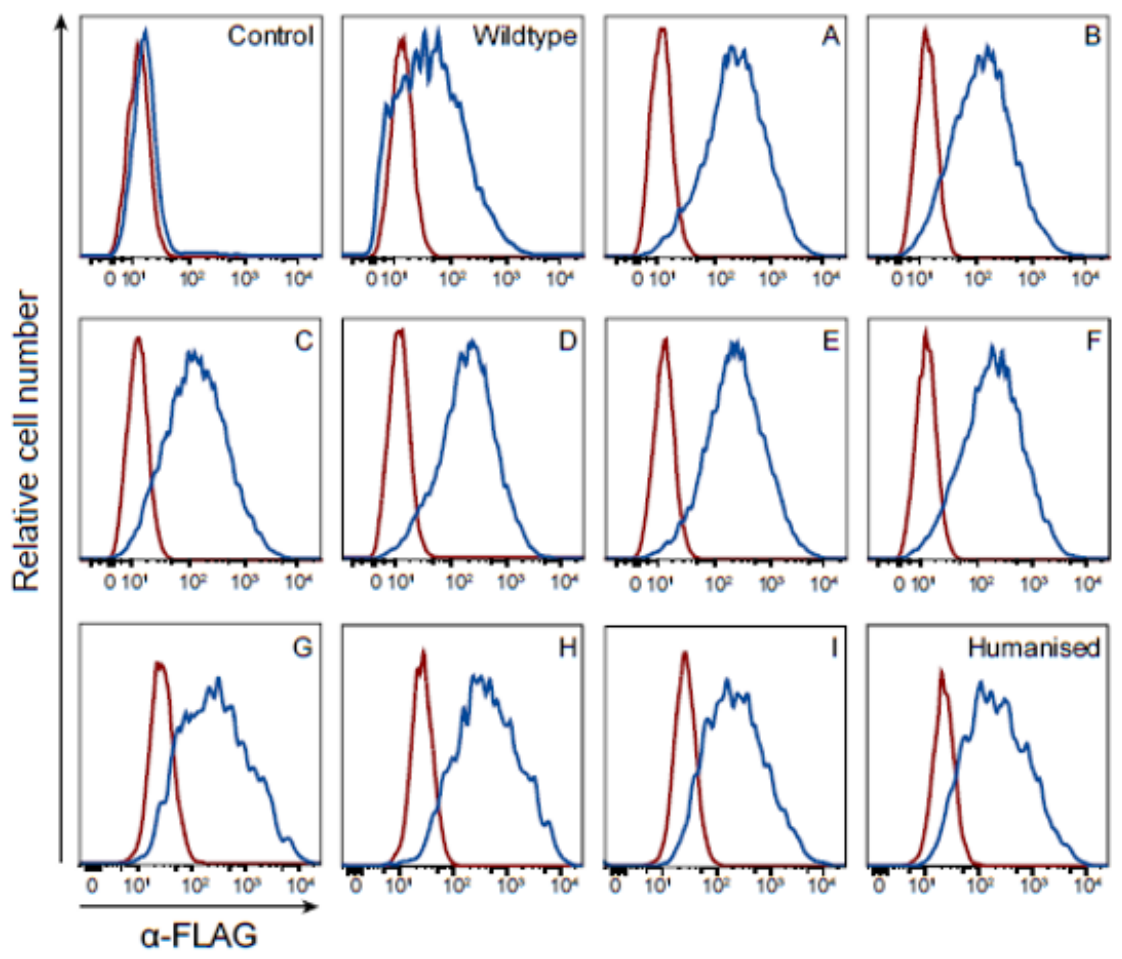

672 Supplementary Figure 1. Expression of receptor variants in U937 cells.

673 Expression of FLAG-tagged wild type, partially humanised dreC5aR receptor variants A-I, and

674 dreC5aR with a fully humanised CHIPS binding site ('humanised') on transduced U937 cells

675 was evaluated by flow cytometry following staining with an anti-FLAG antibody and an APC-

676 conjugated secondary antibody (blue). Control, empty-vector transduced U937 cells. Staining

677 without the primary antibody (red) was included as negative control. 
A

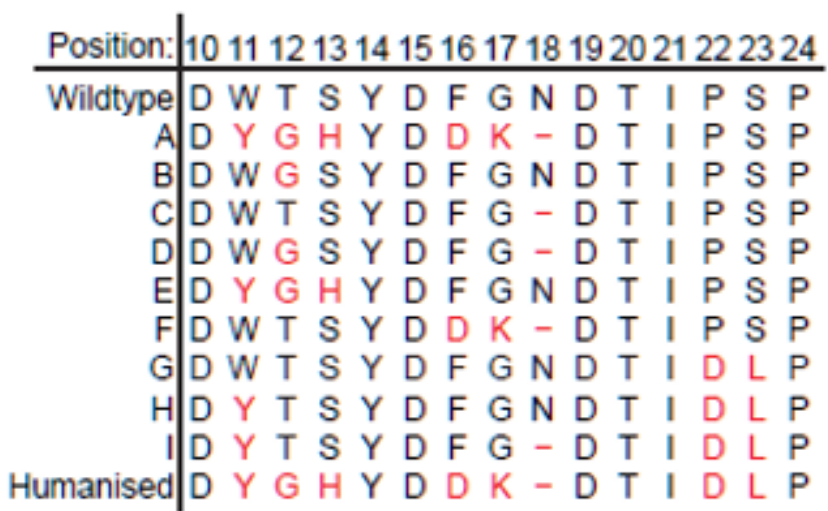

B
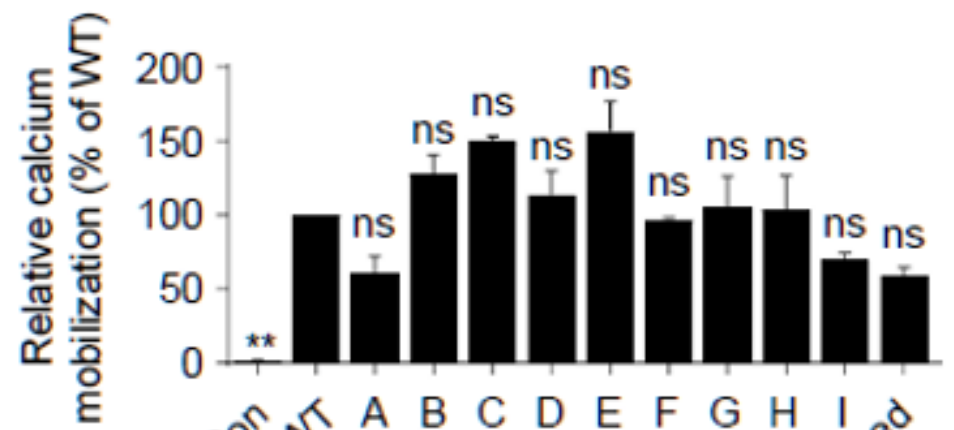

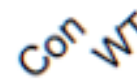

A B C D E F G H

C
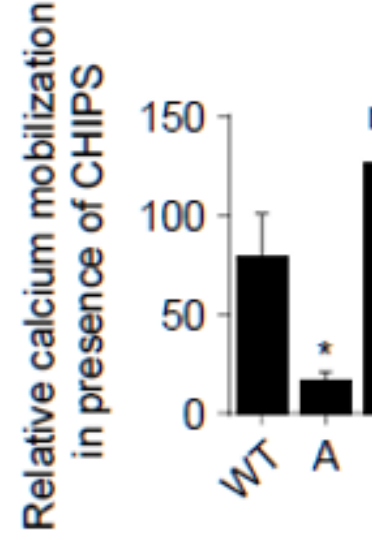

ns

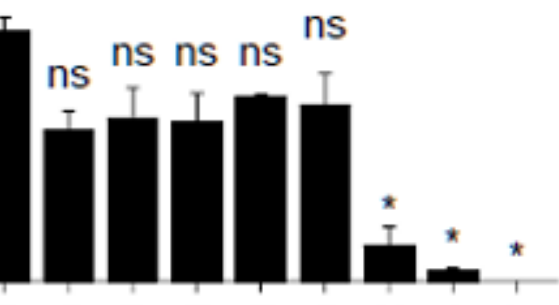

B C D E F G H

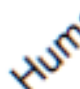

680

681 Supplementary Figure 2. Three amino-acid changes in the CHIPS binding site of

682 dreC5aR confer susceptibility to inhibition by CHIPS.

683 A) Overview of the ten dreC5aR (A-I and fully humanised) variants with partially humanised

$684 \quad \mathrm{~N}$-termini tested; altered amino acids indicated in red, numbers refer to amino acid positions

685 in the dreC5aR. B) Relative C5aR-signalling activity in U937 cells stably expressing an empty

686 vector control (Con), the wild type dreC5aR (WT), or the dreC5aR variants described in (A); 
687 assessed by measuring calcium mobilisation by flow cytometry using the fluorescent probe 688 Fluo-3AM following incubation with $3.3 \mu \mathrm{M}$ recombinant His-dreC5a. Data is presented as 689 relative fluorescent signal compared to the wild type U937-dreC5aR (WT, set at 100\%). C) $690 \mathrm{C} 5 \mathrm{aR}$ activity determined as in (B) following incubation with $3.3 \mu \mathrm{M}$ recombinant His-dreC5a 691 in the absence or presence of $10 \mu \mathrm{g} / \mathrm{ml}$ recombinant CHIPS; presented as percentage 692 fluorescent signal of the CHIPS-treated sample relative to the CHIPS-negative control. All data 693 are presented as means \pm SD and were analysed using a two-way ANOVA with Dunnett's 694 multiple comparisons correction; *,$p<0.05 ;{ }^{* *}, p<0.01$; ns, not significant. 


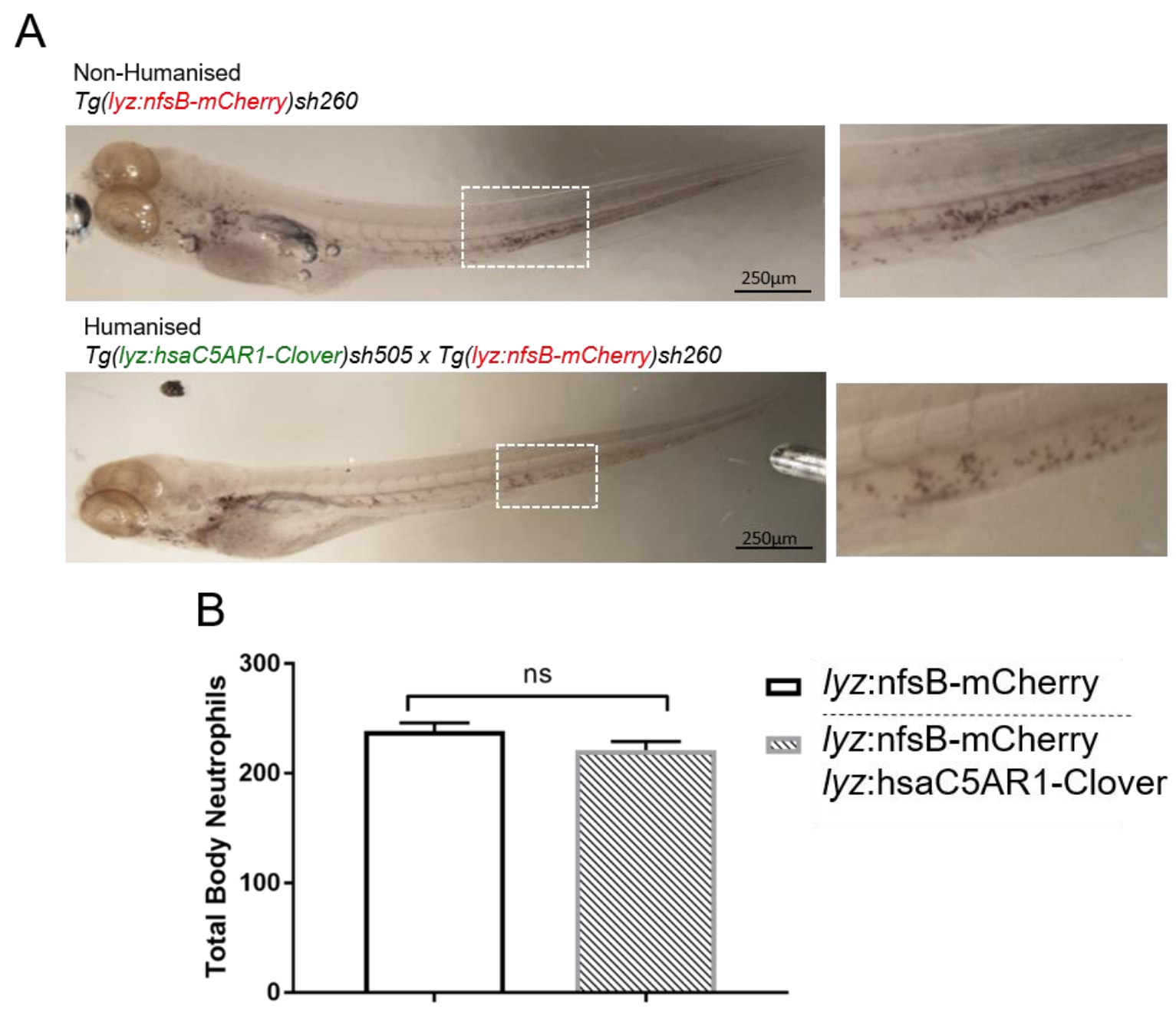

697 Supplementary Figure 3. Expression of the human C5a receptor does not interfere 698 with zebrafish haematopoiesis.

699 A) 4dpf larvae from a $T g(l y z: h s a C 5 A R 1-C l o v e r) s h 505 \times T g(l y z: n f s B-m C h e r r y) s h 260$ cross, 700 separated into non-humanised (lyz:nfsB-mCherry only) and humanised (lyz:hsaC5AR1701 Clover; lyz:nfsB-mCherry) groups and stained with Sudan Black B to detect neutrophils. The 702 white dashed box indicates the enlarged view alongside. B) Total body neutrophil counts from 703 both groups. Values shown are mean \pm SEM ( $n=50$ over two experiments); groups were 704 analysed using an unpaired $t$-test (two-tailed). ns, not significant. 
A.
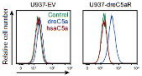

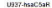

B

FITC

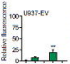

rechaclse - +..

Fen dier.5A - + +

human earer - + +

aebrafsh senm - . +

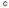

LES7-urecseA

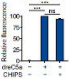

D

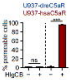

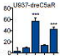

$-+\cdots$

$-+\cdots$

$-\cdots+1$

$-+-\infty$

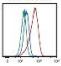

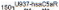

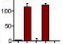

$++-\infty$

$-++-$

- + +

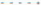

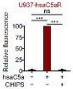

F

1ISA-TARSaR. vog7-loacson

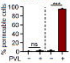




\section{Brightfield}

\section{Clover}

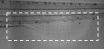

\section{mcherry}

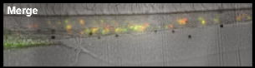

byzhoodsafi-Clover

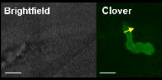

bctrifs-mchery

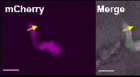

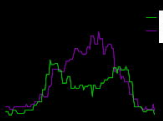

Clover mcherry 

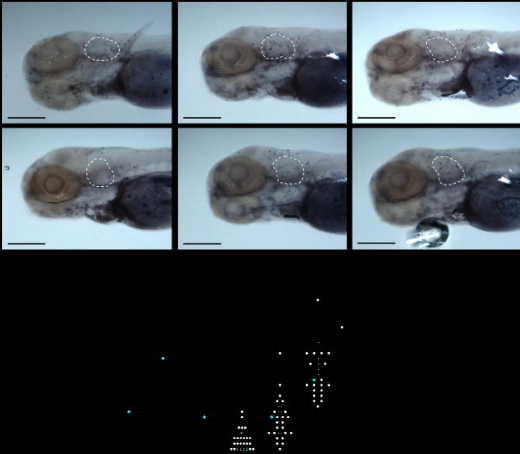

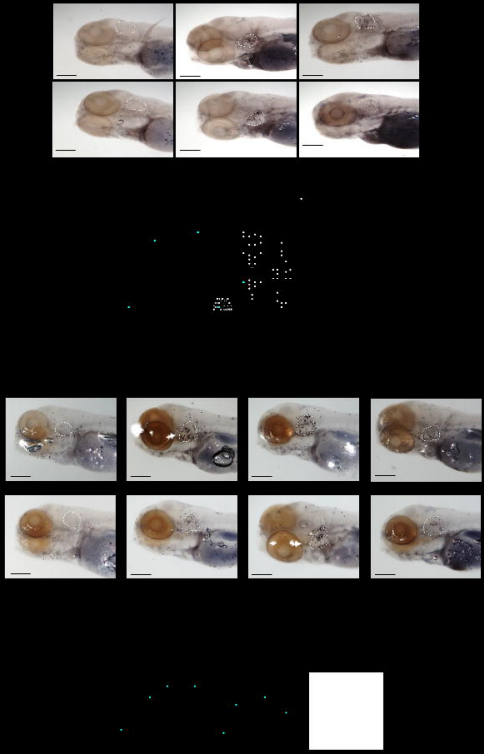

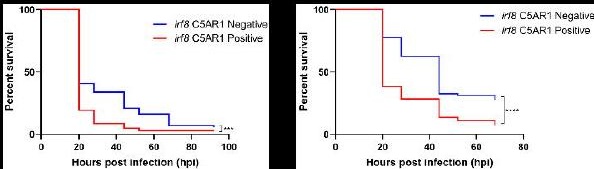